\title{
A neurophysiological insight into use of repetitive transcranial magnetic stimulation as potential therapeutic tool in Parkinson's disease
}

\author{
Giorgio Fuggetta $^{1 *}$, Marco Sandrini $^{1}$, Chiara Arcaro $^{2}$, Michele Tinazzi $^{2}$, Paolo Manganotti $^{2,3}$
}

Parkinson's disease (PD) is a neurodegenerative condition characterised by motor symptoms. In addition, PD patients show pronounced slowing of resting-state oscillatory brain activity compared to healthy controls. Potential therapeutic effects on motor symptoms of PD have been demonstrated by repetitive transcranial magnetic stimulation (rTMS). However, the precise electrophysiological mechanisms behind these effects are unknown. In this cross-over, sham-controlled study, 15 off-drug PD patients underwent both active and sham high-frequency rTMS at $10 \mathrm{~Hz}$ performed on the primary motor cortex (M1). Active rTMS improved overall motor performance and induced an increase of oscillatory activity with a shift of low electroencephalogram (EEG) alpha $(\alpha)$ peak towards higher frequencies of around 9.00-9.75 Hz 30 minutes after active brain stimulation. This pattern of brain rhythm modulation suggests that $10 \mathrm{~Hz}$ rTMS over M1 acts on the thalamocortical resonance interplay entraining neural oscillations at approximately the same frequency, in association with clinical improvement of motor performance in PD.

Keywords: resting-state neural oscillations, brain stimulation, pathophysiology, Parkinson's disease, entrainment of oscillations, neuromodulation

\section{INTRODUCTION}

The abnormal synchronisation and alteration in neural network oscillations between cortical and deep brain structures underlie the pathophysiology of various brain disorders including Parkinson's disease (PD) (1-6). In particular, it has been demonstrated that moderately advanced PD patients without dementia show pronounced slowing of resting-state oscillatory brain activity compared to healthy controls, consisting of a widespread increase in theta $(\theta)$ and low alpha $(\alpha)$ relative power values with a slight change in the cortical distribution of high $\alpha$ power (7).

Transcranial magnetic stimulation (TMS), with rapidly oscillating magnetic fields administered by a coil positioned on the scalp, allows non-invasive stimulation of the human brain (8). It is well documented that repetitive TMS (rTMS) protocols do not pose significant risks in the general population or PD patients $(9,10)$. Moreover, rTMS has been shown to have a therapeutic role in improving symptoms of various neurological and psychiatric disorders (11-14). Of all the movement disorders, PD has received the most attention with regard to rTMS therapeutic studies (11). Several studies have indicated that patients with PD have cortical dysfunction (15). An extensive evidence-based synthesis of established and potential therapeutic applications of rTMS in the neurological and psychiatric domains (12) gave a recommendation of possible efficacy for the effect of highfrequency rTMS $(5-25 \mathrm{~Hz})$ over bilateral (multiple) M1 areas on motor symptoms of PD but no recommendation for highor low-frequency rTMS over unilateral M1 representation of the hand. However, where no recommendation has been proposed, the absence of evidence should not be taken as evidence for an absence of effect. This is especially true for treatment options with highly variable individual responses, such as responses to rTMS (12).

rTMS can be used in basic research to study how perturbations in activity in a focal brain area affect neural network oscillations (16, 17), as it has been shown to interact with spontaneous oscillatory rhythms existing in the cortical circuits (18-22). This may induce

\footnotetext{
${ }^{1}$ Department of Psychology, University of Roehampton, London, SW15 4JD, UK ${ }^{2}$ Department of Neurological and Movement Sciences, University of Verona, Verona, 37134, Italy. ${ }^{3}$ Clinica Neurologica, Azienda Ospedaliera Universitaria di Trieste, University of Trieste, Trieste, 34149, Italy.

${ }^{*}$ Corresponding author. Email: giorgio.fuggetta@roehampton.ac.uk (G.F.)
}

an activity-dependent modulation according to the phase-locking synchrony between cortical oscillations and patterns of the stimulation (20). Interestingly, the modification of cortical oscillatory activity through the use of rhythmic repetitive stimulation $(23,24)$ may readjust pathological patterns of brain activity. This would allow an opportunity to induce new oscillatory patterns, which could adequately modulate the neural response of a network (i.e. entrainment) (25). Modulating these rhythms with optimallydesigned, closed-loop stimulation techniques could be of highly valuable therapeutic potential (26). It is also tempting to consider that the frequency- and pattern-dependent therapeutic effects of rTMS could come, at least in part, from an interaction with some altered oscillations involving cortical networks (20).

The general purpose of this electrophysiological study was to enhance the understanding of the probable electrophysiological mechanism underlying the therapeutic effects of rTMS in PD patients. To achieve this, the effect of rTMS induced modulation of oscillatory activity was compared with the alteration of oscillations in PD. The hypothesis that a transient effect of modulation of $\alpha$ band oscillations $(8-12 \mathrm{~Hz})$ in PD patients at rest could be externally entrained by rTMS at a frequency of $10 \mathrm{~Hz}$ was tested. The resolution of this matter would be of considerable interest because it could suggest new ways to use combined EEG/TMS techniques as both a diagnostic test and a therapeutic tool to restore proper neural oscillations in PD. To address this issue, we used surface EEG to quantify the cortical oscillatory activity pre- and post-rTMS over M1.

Several lines of evidence support the use of the aforementioned frequency of rTMS in the current study. Reviews of clinical trials which assessed the therapeutic effects of rTMS in PD have demonstrated stronger beneficial effects of high-frequency compared to low-frequency rTMS over M1 on motor symptoms in PD patients $(11,12,27)$. Furthermore, a series of studies using positron emission tomography (PET) provide evidence that short trains of $10 \mathrm{~Hz}$ rTMS could stimulate subcortical dopamine release in the striatum (specifically, the caudate nucleus and putamen) in healthy participants and PD patients $(28-31)$. The release of dopamine in the putamen was observed to be greater in the more affected hemisphere in mild hemiparkinsonian patients (29). It has 
also been demonstrated that rTMS at $10 \mathrm{~Hz}$ could act indirectly on the subcortical level via stimulation at connected cortical areas (29). Additionally, the short interpulse interval of highfrequency stimulation could build up a large "summation" of neural activity which would consequently result in greater recruitment and activation of the descending corticothalamic pathways $(20,22)$. Taken together, high-frequency rTMS at $10 \mathrm{~Hz}$ shows a promising potential to act on the abnormal processing in the corticobasal ganglial loops which underlies PD symptoms (11).

\section{RESULTS}

No adverse side effects of rTMS were reported by any of the participants during the experiment. Results of the two interventions - $10 \mathrm{~Hz}$ active and sham rTMS - are shown in Fig. 1 and Fig. 2, displaying changes in motor performance score and relative EEG power (reflecting the regional oscillatory activity of neural assemblies) respectively.

\section{Clinical motor performance}

A two-way "rTMS condition" $\mathrm{x}$ "time of evaluation" repeatedmeasures ANOVA was conducted to evaluate the effects of rTMS on the overall motor performance in PD patients. There was a significant main effect of "time of evaluation" $\left(F_{(1,14)}=22.9, p<.001\right.$, $\eta p 2=.62$ ), revealing an overall decrease in UPDRS-III (motor subscale) score after rTMS intervention (pre-stimulation: 14.0; 35 minutes post-stimulation: 10.5), indicating significant improvement. There was also a significant two-way "rTMS condition" $\mathrm{x}$ "time of evaluation" interaction $\left(F_{(1,14)}=37.7, p<.001, \eta p 2=.73\right)$. Interestingly, post-hoc comparisons showed that both rTMS conditions improved overall motor performance. In particular, active rTMS was associated with a $35.4 \%$ improvement of motor response (pre-stimulation: $13.93 ; 35$ minutes post-stimulation: $9.00 ; p<.001$ ) and sham rTMS a $14.6 \%$ improvement (pre-stimulation: $14.13 ; 35$ minutes post-stimulation: $12.07 ; p<.01$ ). Moreover, improvement in overall motor performance measured 35 minutes after stimulation was significantly more pronounced following active rTMS than sham rTMS (9.00 and 12.07 respectively; $p<.01$, Bonferronicorrected). Fig. 1 shows mean total UPDRS-III (motor subscale) scores before and 35 minutes after rTMS intervention.

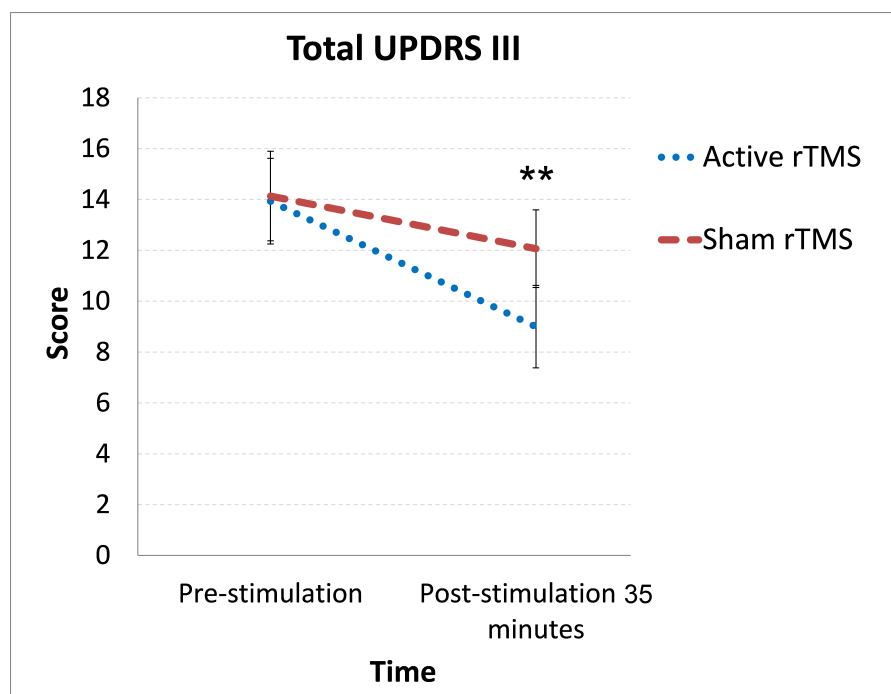

Fig. 1. Mean total UPDRS-III (motor subscale) score ( \pm SEM) before and after active and sham rTMS intervention. ${ }^{* *} p<.01$; Bonferroni corrected.
Table 1. ANOVA statistical results of relative EEG power for frontal, central, parietal and occipital electrodes at $\alpha(8.00-12.75 \mathrm{~Hz})$ frequency ban

\begin{tabular}{|c|c|c|c|c|}
\hline Effect & Frontal & Central & Parietal & Occipital \\
\hline Time & $\begin{array}{l}F(2,28)=4.3,{ }^{*} \\
\eta 2 \mathrm{p}=.24\end{array}$ & $\begin{array}{l}F(2,28)=1.4, p= \\
\text { ns, } \eta 2 p=.09\end{array}$ & $\begin{array}{l}F(2,28)=5.3, * \\
\eta 2 \mathrm{p}=.27\end{array}$ & $\begin{array}{l}F(2,28)=2.3, * \\
\eta 2 \mathrm{p}=.14\end{array}$ \\
\hline rTMS Condition & $\begin{array}{l}F(1,14)=0.0, p= \\
\text { ns, } \eta 2 \mathrm{p}=.00\end{array}$ & $\begin{array}{l}F(1,14)=0.2, p= \\
\text { ns, } \eta 2 p=.02\end{array}$ & $\begin{array}{l}F(1,14)=0.2, p= \\
\text { ns, } \eta 2 \mathrm{p}=.01\end{array}$ & $\begin{array}{l}F(1,14)=0.0, p= \\
\mathrm{ns}, \eta 2 \mathrm{p}=.00\end{array}$ \\
\hline Frequency Bin & $\begin{array}{l}F(1.7,24.5)=6.9 \\
* *, \eta 2 \mathrm{p}=.33\end{array}$ & $\begin{array}{l}F(1.7,23.6)=5.8 \\
*, \eta 2 \mathrm{p}=.29\end{array}$ & $\begin{array}{l}F(2.0,28.2)=4.3 \\
*, \eta 2 \mathrm{p}=.23\end{array}$ & $\begin{array}{l}F(1.8,25.9)=6.0 \\
* *, \eta 2 \mathrm{p}=.30\end{array}$ \\
\hline $\begin{array}{l}\text { Time * rTMS } \\
\text { Condition }\end{array}$ & $\begin{array}{l}F(1.2,16.5)=0.1 \\
p=\mathrm{ns}, \eta 2 \mathrm{p}=.01\end{array}$ & $\begin{array}{l}F(1.2,17.2)=0.1 \\
p=\mathrm{ns}, \eta 2 \mathrm{p}=.01\end{array}$ & $\begin{array}{l}F(2,28)=0.1, p= \\
\text { ns, } \eta 2 p=.01\end{array}$ & $\begin{array}{l}F(1.2,16.6)=0.0 \\
p=\mathrm{ns}, \eta 2 \mathrm{p}=.00\end{array}$ \\
\hline $\begin{array}{l}\text { Time * } \\
\text { Frequency bin }\end{array}$ & $\begin{array}{l}F(3.4,48.4)=2.8 \\
*, \eta 2 \mathrm{p}=.16\end{array}$ & $\begin{array}{l}F(3.5,48.8)=1.4 \\
p=\mathrm{ns}, \eta 2 \mathrm{p}=.09\end{array}$ & $\begin{array}{l}F(3.6,51.0)=1.4 \\
p=\mathrm{ns}, \eta 2 \mathrm{p}=.09\end{array}$ & $\begin{array}{l}F(4.1,57.9)=3.1, \\
*, \eta 2 \mathrm{p}=.18\end{array}$ \\
\hline $\begin{array}{l}\text { Condition * } \\
\text { Frequency bin }\end{array}$ & $\begin{array}{l}F(2.3,32.3)=0.5 \\
p=\mathrm{ns}, \eta 2 \mathrm{p}=.04\end{array}$ & $\begin{array}{l}\mathrm{F}(2.0,28.5)=0.5 \\
p=\mathrm{ns}, \eta 2 \mathrm{p}=.03\end{array}$ & $\begin{array}{l}F(4,56)=0.1, \mathrm{p}= \\
\mathrm{ns}, \eta 2 \mathrm{p}=.00\end{array}$ & $\begin{array}{l}F(2.5,35.6)=0.2 \\
p=\mathrm{ns}, \eta 2 \mathrm{p}=.02\end{array}$ \\
\hline $\begin{array}{l}\text { Time * rTMS } \\
\text { Condition* } \\
\text { Frequency Bin }\end{array}$ & $\begin{array}{l}F(3.1,42.8)=0.5 \\
p=\mathrm{ns}, \eta 2 \mathrm{p}=.07\end{array}$ & $\begin{array}{l}F(2.7,38.1)=3.4 \\
*, \eta 2 p=.20\end{array}$ & $\begin{array}{l}F(4.3,60.8)=2.1 \\
p=\mathrm{ns}, \eta 2 \mathrm{p}=.13\end{array}$ & $\begin{array}{l}F(3.1,43.9)=0.8 \\
p=\mathrm{ns}, \eta 2 \mathrm{p}=.05\end{array}$ \\
\hline
\end{tabular}

$\mathrm{ns}=$ non-significant. Significant 3-way interaction is indicated in bold. ${ }^{*} p<.05,{ }^{* *} p<.01$.

\section{Relative EEG power at $\alpha(8.00-12.75 \mathrm{~Hz})$ frequency band}

Table 1 shows a summary of ANOVA statistical results of relative EEG power for frontal, central, parietal and occipital electrodes at $\alpha(8.00-12.75 \mathrm{~Hz})$ frequency band.

A 3-way "rTMS condition" x "time of evaluation" x "frequency bin" repeated-measures ANOVA was conducted to examine the effects of rTMS on regional oscillatory activity (as reflected by relative EEG power) for each of the four electrode sites: frontal (F3/F4), central (C3/C4), parietal (P3/P4) and occipital (O1/O2). Significant 3-way "rTMS condition" $\mathrm{x}$ "time of evaluation" $\mathrm{x}$ "frequency bin" interaction was only observed at the central electrode site. As a baseline check, comparison between active and sham rTMS conditions revealed no pre-stimulation significant difference between the two rTMS conditions at the central electrode site for the entire $\alpha$ frequency band of interest $(8.00-12.75 \mathrm{~Hz})$. Posthoc comparisons following the significant interaction indicated a significant increase in regional oscillatory activity (as reflected by a significant increase in relative EEG power) across the first and the final evaluation time points (pre-stimulation: $7.0 \% ; 30$ minutes post-stimulation: $10.5 \% ; p<.05$; Bonferroni-corrected) and across the final two evaluation time points (immediately post-stimulation: $7.2 \%$; 30 minutes post-stimulation: $10.5 \%$; $p<.05$; Bonferronicorrected) in the active rTMS condition at frequency bin 9.00$9.75 \mathrm{~Hz}$ (see Fig. 2). This was the only condition where such an increase was observed; no other significant modulation of cortical oscillations was noted following either active or sham rTMS across any of the other frequency bins. In other words, active but not sham rTMS stimulation induced a modulation of oscillatory activity with a shift of dominant frequency $\alpha$ peak towards higher frequencies of around 9.00-9.75 Hz at the central electrode site (Fig. 2).

\section{DISCUSSION}

The general purpose of this study was to gain new insight into a probable electrophysiological mechanism underlying the potential therapeutic effects of rTMS in PD patients. In particular, we aimed to evaluate if brain stimulation with TMS could readjust pathological patterns of brain oscillatory activity which is alterated in PD patients $(7,32)$. The main finding of the current study indicated that excitatory rTMS at $10 \mathrm{~Hz}$ induced a specific 

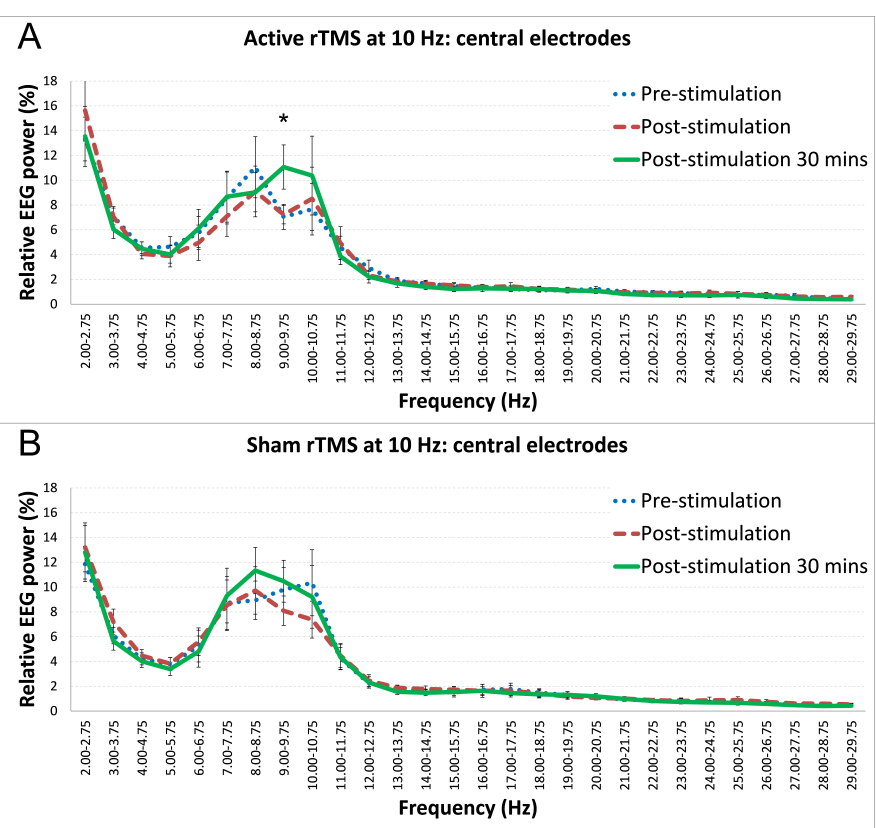

Fig. 2. Superimposition of mean ( \pm SEM) relative power transformation from 2.00 to $29.75 \mathrm{~Hz}$ averaged over measurements recorded by the central electrodes. Active but not sham rTMS at $10 \mathrm{~Hz}$ induced a power increase 30 minutes after brain stimulation with a shift of dominant frequency $\alpha$ peak towards higher frequencies of around 9.00-9.75 Hz. ${ }^{*} p<.05$; Bonferroni corrected.

increase of amplitude in low $\alpha$ relative power with a shift of the dominant frequency $\alpha$ peak towards higher frequencies of around $9.00-9.75 \mathrm{~Hz}$ in PD patients 30 minutes post stimulation. This direct electrophysiological modulation of cortical oscillatory activity was concomitant with improvement in clinical motor symptoms observed in the same group of patients.

Data published to date suggest possible anti-parkinsonian effects of non-invasive rTMS on motor symptoms, especially when applied at high frequency on large M1 regions of both hemispheres. The majority of studies show global improvement in the motor subscale of UPDRS, especially in movement speed or gait velocity, following focal stimulation of hand representation in the brain (12). In particular, a small number of single session, proof-of-principle studies have shown a $10-30 \%$ improvement of clinical motor symptoms following active but not sham rTMS (11). The improvement of motor performance of the current study was $20.8 \%$ greater in the case of active rTMS compared to sham rTMS over the motor cortex in PD. This is consistent with findings of previous clinical studies.

A number of investigations have demonstrated that the rhythmic aspects of non-invasive TMS over M1 can induce modification of cortical oscillatory activity (18-22). In particular, high-frequency stimulation $(>5 \mathrm{~Hz})$ leads to an immediate synchronisation of EEG activity with an increase of power particularly in the $\alpha$ band, consistent with entrainment of oscillatory activity $(21,22)$. By using concurrent EEG in PD patients, our results show direct evidence for causal entrainment of brain oscillations by rTMS, consistent with previous findings. In the current study, significant rTMS aftereffects emerged 30 minutes after the end of the stimulation trains. Recently, some molecular mechanisms have been postulated to explain the slow building up (generally in the region of 510 minutes) of rTMS-induced aftereffects in humans (33-35). Research investigating the pathophysiology of PD mostly focuses on basal ganglia dysfunction. However, the main output from the basal ganglia is via the thalamus and corticothalamic feedback constitutes the primary source of synapses in the thalamus (4). We therefore focused on the thalamocortical interplay to explain the results of the current proof-of-principle study.

A pathophysiological framework based on Thalamocortical Dysrhythmia (TCD) has been proposed by Rodolfo Llinas to explain the alteration of brain oscillations common across PD, neurogenic pain, epilepsy, tinnitus and major depression (3). In the TCD phenomenon, bursting thalamic relay neurons exert a rhythmic influence on thalamocortical modules in the $\theta$ frequency band (36). This disrupts the normal, state-dependent flow of information between the thalamus and the cortex (37), leading to disturbance of sensation, motor performance and cognition. The existence of TCD has been demonstrated in patients at rest in studies employing various different methodologies including magnetoencephalography (MEG), EEG and single cell physiology (2-6). The pathophysiological framework of TCD has been adopted to explain the genesis of parkinsonian symptoms (4). In a groundbreaking study by Sarnthein and Jeanmonod (4), local field potentials (LFPs) were recorded from pallidal-recipient thalamic nuclei in the ventral lateral anterior (VLa) and the ventral anterior (VA) regions during a surgical intervention in PD patients. Simultaneously, EEG was recorded from several sites on the scalp. The highest thalamocortical coherence which reached $70 \%$ was found in the $\theta$ frequency band $(4-9 \mathrm{~Hz})$ with a mean peak frequency of $7.5 \mathrm{~Hz}$ at the frontal scalp sites on both hemispheres. The high thalamocortical coherence underlines the importance of thalamic function for the genesis of scalp EEG (4). The current findings are supporting the various pieces of evidence in favour of proposing the use of cortical stimulation in the treatment of PD (26). In particular, our findings are consistent with existing research suggesting that the modification of cortical oscillations through the use of noninvasive brain stimulation may have therapeutic effects of restoring proper neural oscillations in PD patients (20).

Overall, the current electrophysiological results suggest that targeting the specific cortical areas in the most affected hemisphere by brain stimulation could improve the cortical dysfunction in PD directly or modify activity in the basal ganglia networks with corticostriatal and corticosubthalamic projections (38). Indeed, the use of $10 \mathrm{~Hz}$ rTMS in the present study appears to be able to modulate the rhythmic thalamocortical interplay by entraining the resonance between the thalamus and the cortex at approximately the same frequency $(\sim 9.00-9.75 \mathrm{~Hz})$, thus generating a state that seems to restore proper neural oscillations in association with a clinical improvement of motor performance in PD. Such improvement could be related to an increase in dopamine release, although the slight improvement of motor symptoms after sham rTMS also suggests the possibility of a partial intervention of placebo effects in PD as in previous studies $(29,39)$. Nevertheless, after controlling for the placebo and auditory effects with the sham rTMS condition, we demonstrated that active rTMS was capable of acting on internally generated and persistent thalamic frequency range activity and served as a trigger to temporally re-establish proper thalamocortical resonance oscillatory network function, with a consequent increased $\alpha$ rhythmicity in PD patients at rest 30 minutes after stimulation. These results support the hypothesis that brain stimulation techniques such as TMS can trigger an oscillation and reset the ongoing rhythmic activity of a local thalamic pacemaker $(20,28,40)$. Once oscillations are reset, regionally-specific endogenous rhythms of the brain may reemerge, restoring normal brain function (41). Thus, the cortical targets of non-invasive brain stimulation might be conceptualised as 
"entry ports" for modulation of activity in specific bi-hemispheric, cortico-subcortical neural networks (38).

Some limitations of the current study should be mentioned. First, given that our sample size was relatively small, findings reported here should be reproduced in larger cohorts before firm conclusions could be drawn. Second, the sham condition in the within-participants design employed by the current study was insufficient to demonstrate the specificity of TMS effects (42). Third, the absence of a control stimulation condition (i.e. using a different stimulation frequency) meant that nonspecific effects of the stimulation could not be ruled out.

In conclusion, results of the present investigation point to the potential of rTMS as a therapeutic tool to reverse the pathological slowing of oscillatory brain activity observed in Parkinson's disease. By examining the effects of rTMS within the framework of dysrhythmic thalamocortical interplay, this investigation provides insight into the mechanism underlying potential therapeutic effects of rTMS and sheds light on possible sensitive treatment efficacy measures based on EEG oscillation indexes (20). Future work could examine changes of multi-band EEG power across various brain regions with more advanced data analysis methods in order to provide more comprehensive evidence. Future EEG studies, using an increased number of electrodes, would allow accurate source location analysis which could further our understanding of brain oscillations with reference to the thalamocortical loop. Future applied clinical research could attempt to further develop therapeutic strategies to restore of normal oscillatory patterns in association with effective treatment of PD. Specifically, future research could employ multiple stimulation sessions (e.g. one session per day for a week) to examine the clinical improvements and neural changes.

\section{MATERIALS AND METHODS \\ Participants}

A total of 16 patients were initially recruited for this cross-over, sham-controlled study. However, one patient was excluded due to failure in obtaining a reliable determination of resting motor threshold. Thus, 15 patients (aged 45-80 years) with idiopathic Parkinson's disease (disease duration of 2-13 years) were included in this study. Level of cognitive impairment was determined using the Mini Mental State Examination (MMSE) (43). Disease duration was estimated from patients' subjective report of first occurrence of motor symptoms. Side of onset was determined by the bodyhalf in which these symptoms first occurred. Motor performance score and PD stage were obtained using the motor subscale of the Unified Parkinson's Disease Rating Scale (UPDRS-III) (44) and the Modified Hoehn and Yahr Scale (45) 12 hours after an overnight withdrawal of anti-parkinsonian medication, i.e. in an "off-drug" state, by a trained physician prior to and after rTMS sessions. Characteristics of patients are listed in Table 2. Written informed consent was obtained from all participants and ethical approval was provided by the Medical School Ethics Committee of Verona University, Italy. Ethics review criteria conformed to the Declaration of Helsinki.

\section{Experimental paradigm}

Participants were tested in a quiet and dimly lit room. An initial motor performance evaluation was assessed clinically using the UPDRS-III. Participants were then seated in a comfortable armchair and were instructed to remain at rest and at an awake state. An EEG cap was mounted, with impedance check performed. TMS parameter of the individual's resting motor threshold (RMT) was measured (see below). Consistent with previous studies, patients were instructed to keep their eyes closed during EEG recordings - from two minutes before until 30 minutes after the end of rTMS delivery - in order to maximise occipital $\alpha$ oscillations and their influence on the thalamocortical circuit $(3,46)$. One of the two interventions, i.e. active or sham rTMS at $10 \mathrm{~Hz}$ (see below), was performed on the hand motor area of the primary motor cortex (M1) contralateral to the most affected body side while EEG activity was recorded simultaneously and continuously. The EEG cap was removed from the patients 30 minutes after rTMS intervention. Thirty-five minutes after the end of the rTMS intervention, a clinician performed motor evaluation with the UPDRS-III again. The order of the two rTMS sessions was randomised across patients and the two sessions were separated by 48 hours in order to minimise carryover effects. The duration of rTMS effects on motor symptoms is considered to last less than one hour after a single rTMS intervention (11). All patients were naive to rTMS prior to the study and were unfamiliar with the differences between active and sham rTMS with regard to acoustic and tactile artefacts. Twenty intermittent trains of 40 pulses (800 stimuli) were delivered during each of the two rTMS conditions at $10 \mathrm{~Hz}$. The duration of each train was of 4 seconds and the intertrain interval (ITI) was 30 seconds. The total duration of rTMS was 10 minutes. The intensity of the two rTMS conditions was set at $10 \%$ below the individual's RMT. The motor threshold, which reflects the global excitability of the corticospinal motor pathway, has been defined as the intensity of TMS that produces an identifiable MEP of $\sim 50 \mu \mathrm{V}$ in at least five out of ten TMS pulses (47).

Table 2. Characteristics of Participants.

\begin{tabular}{ll}
\hline & $\begin{array}{l}\text { Patients with } \\
\text { Parkinson's } \\
\text { disease }(\mathrm{N}=15)\end{array}$ \\
\hline Age (years, mean \pm SD) & $64.1 \pm 12.6$ \\
Gender ( $ð /$ ) & $13 / 2$ \\
MMSE (mean \pm SD) & $27.7 \pm 2.8$ \\
Side of Onset (Left/Right) & $9 / 6$ \\
Disease Duration (Years; Mean \pm SD) & $4.9 \pm 3.4$ \\
H and Y Modified “OFF” (1/1.5/2/2.5/3) & $2 / 5 / 4 / 1 / 3$ \\
UPDRS-III “OFF” (mean \pm SD) & $14.0 \pm 6.5$ \\
Stimulation Side (Left/Right) & $8 / 7$
\end{tabular}

MMSE = Mini Mental State Examination; $\mathrm{H}$ and Y Modified = Modified Hoehn and Yahr Scale; UPDRS-III = Unified Parkinson's Disease Rating Scale (motor subscale); "OFF" = Off-drug

\section{Transcranial magnetic stimulation (TMS)}

TMS, performed using a high-power Magstim-Rapid stimulator (Magstim, Whitland, UK), was delivered through a figure-of-eight coil (70 mm standard coil; Magstim), oriented so that the induced electric current flowed in a posterior-to-anterior direction over the motor cortex. The coil was placed tangentially to the scalp with the handle pointing backwards and laterally at a $45^{\circ}$ angle away from the midline perpendicular to the line of the central sulcus to achieve the lowest motor threshold. rTMS was applied over the left or right primary motor cortex (M1), in the proximity of the $\mathrm{C} 3$ or $\mathrm{C} 4$ 
electrode, simultaneously with EEG data collection. A sham rTMS condition was carried out to control for the air- and bone-conducted auditory stimuli that could confound EEG oscillations in the target motor system. The sham rTMS condition was performed with the coil tilted at $90^{\circ}$ to the skull in order to avoid real stimulation of the motor cortex.

Motor Evoked Potentials (MEPs) were recorded from the left or right thenar eminence (TE) muscle using $\mathrm{Ag} / \mathrm{AgCl}$ surface electrodes in a belly-tendon montage. The amplified and bandpassfiltered $(50 \mathrm{~Hz}-5 \mathrm{kHz})$ electromyogram (EMG) signal was fed into a Micromed machine (Micromed, Treviso, Italy). Optimal position for TE activation was determined by moving the coil in $0.5-\mathrm{cm}$ steps around the hand area of the motor cortex of the most affected hemisphere. The optimal position was defined as the site where stimuli of slightly suprathreshold intensity consistently produced the largest MEPs with the steepest negative slope in the target muscle, referred to as "motor hotspot".

\section{Electroencephalographic (EEG) acquisition and analysis}

Continuous EEG was recorded with a MR compatible EEG amplifier (SD MRI 32, Micromed, Treviso, Italy). The EEG signal was recorded from $19 \mathrm{Ag} / \mathrm{AgCl}$ electrode sites. Electrode montage and placement were performed according to the International 10-20 system. Consistent with previous studies using the same system, the reference electrode was positioned at $\mathrm{AFz}$ site, whereas the ground electrode was placed at $\mathrm{FCz}$ site $(21,28)$. The impedance was kept below $10 \mathrm{k} \Omega$. The activities in the right TE muscle and in the right eye vertical electroculogram (vEOG) were bipolarly registered from two surface EMG electrodes. To ensure safety, the wires were carefully arranged to avoid loops and physical contact with the participants. To avoid electrical saturation of EEG channels induced by TMS, the EEG amplifier had a resolution of 22 bits with a range of $\pm 25.6 \mathrm{mV}$. An anti-aliasing hardware bandpass filter was applied with a bandwidth between 0.15 and $269.50 \mathrm{~Hz}$. EEG data were sampled at a frequency of $1024 \mathrm{~Hz}$ using SystemPlus (Micromed, Treviso, Italy).

A re-reference off-line was obtained by the application of reference electrode standardisation technique (REST) (48). EEG data were processed off-line using BrainVision Analyzer (Munich, Germany). To demonstrate rTMS effects on modulation of EEG oscillations, the following four pairs of homologous electrodes across hemispheres were selected for analysis: F3/F4, C3/C4, P3/P4 and O1/O2. Data from three time points were used for analysis: (1) 120 seconds immediately before, (2) immediately after and (3) 30 minutes after each 10-minute brain stimulation of 20 intermittent trains of rTMS. Two minutes of continuous data were divided into 30 consecutive, non-overlapping epochs of 4,096 data points. Subsequently, the data were digitally filtered with a bandpass of 1-46 Hz. Epochs with eye movements and muscle or movement artefacts (as indicated by HEOG activity exceeding $\pm 40 \mu \mathrm{V}$ and activity at other electrodes exceeding $\pm 70 \mu \mathrm{V}$ ) were excluded. This resulted in the exclusion of $\sim 7$ epochs per rTMS condition (23\% rejection rate). A discrete Fast Fourier Transform (FFT) of the remaining epochs with a resolution of $0.25 \mathrm{~Hz}$ was computed for all electrodes and then averaged. Non-overlapping hamming-windows controlled spectral leakage. The FFT power value measurements within each frequency between 2.00 and $45.75 \mathrm{~Hz}$ were averaged to create 44 non-overlapping $<1 \mathrm{~Hz}$ frequency bins. The relative power (which reduced the effect of inter-participant variability in absolute power) at each frequency bin was calculated by dividing the absolute power at each bin by the sum of absolute power of all frequency bins over the entire frequency range (2.00-45.75 $\mathrm{Hz}$ ), as in a previous study (32). Using relative power rather than absolute power resulted in a lower variance of power values within participant groups (7). The $\alpha$ frequency band of interest (8.00$12.75 \mathrm{~Hz}$ ), containing five frequency bins, was chosen for statistical analysis.

\section{Statistical analysis}

Data were analysed using SPSS for Windows Version 20.0. First, we examined the effects of rTMS protocol on the overall motor performance in PD patients using the total UPDRS-III (motor subscale) score as the dependent variable in a repeated-measures ANOVA with the following factors: "rTMS condition" (active rTMS and sham rTMS) and "time of evaluation" (pre-stimulation and 35 minutes post-stimulation). Second, to assess whether rTMS artificially induced modulation of neural oscillations in the $\alpha$ frequency range during eyes-closed resting state, mean relative EEG power values were submitted to four separate repeatedmeasures ANOVA for frontal $(\mathrm{F} 3 / \mathrm{F} 4)$, central $(\mathrm{C} 3 / \mathrm{C} 4)$, parietal (P3/P4) and occipital (O1/O2) pairs of electrodes. Each ANOVA had the factors: "rTMS condition" (active rTMS and sham rTMS), "time of evaluation" (pre-stimulation, immediately post-stimulation and 30 minutes post-stimulation) and "frequency bin" (8.00-8.75, 9.00-9.75, 10-10.75, 11.00-11.75 and 12.00-12.75 Hz). For each ANOVA, the sphericity assumption was assessed with Mauchly's test. Greenhouse-Geisser epsilon adjustments for non-sphericity were applied where appropriate. Bonferroni corrections of $p$-values were used for multiple comparisons with post-hoc paired $t$-tests. For all statistical tests, $p<.05$ was considered significant, except where Bonferroni corrections were applied.

\section{REFERENCES}

1. P. Brown, Bad oscillations in Parkinson's disease. J Neural Transm. Suppl. pp. 27-30 (2006).

2. D. Jeanmonod, J. Schulman, R. Ramirez, R. Cancro, M. Lanz, A. Morel, M. Magnin, M. Siegemund, E. Kronberg, U. Ribary, M. Llinas, Neuropsychiatric thalamocortical dysrhythmia: Surgical implications. Thalamus Relat. Syst. 2, 103 (2003).

3. R. R. Llinas, U. Ribary, D. Jeanmonod, E. Kronberg, P. P. Mitra, Thalamocortical dysrhythmia: A neurological and neuropsychiatric syndrome characterized by magnetoencephalography. Proc. Natl. Acad. Sci. U.S.A. 96, 15222-15227 (1999).

4. J. Sarnthein, D. Jeanmonod, High thalamocortical theta coherence in patients with Parkinson's disease. J. Neurosci. 27, 124-131 (2007).

5. J. Sarnthein, D. Jeanmonod, High thalamocortical theta coherence in patients with neurogenic pain. Neurolmage 39, 1910-1917 (2008).

6. J. J. Schulman, R. Cancro, S. Lowe, F. Lu, K. D. Walton, R. R. Llinás, Imaging of thalamocortical dysrhythmia in neuropsychiatry. Front. Hum. Neurosci. 5, 69 (2011).

7. D. Stoffers, J. L. W. Bosboom, J. B. Deijen, E. C. Wolters, H. W. Berendse, C. J. Stam, Slowing of oscillatory brain activity is a stable characteristic of Parkinson's disease without dementia. Brain 130, 1847-1860 (2007).

8. M. Sandrini, C. Umiltà, E. Rusconi, The use of transcranial magnetic stimulation in cognitive neuroscience: A new synthesis of methodological issues. Neurosci. Biobehav. Rev. 35, 516536 (2011).

9. S. Rossi, M. Hallett, P. M. Rossini, A. Pascual-Leone, Safety, ethical considerations, and application guidelines for the use of transcranial magnetic stimulation in clinical practice and research. Clin. Neurophysiol. 120, 2008-2039 (2009).

10. M. VonLoh, R. Chen, B. Kluger, Safety of transcranial magnetic stimulation in Parkinson's disease: A review of the literature. Parkinsonism Relat. Disord. 19, 573-585 (2013).

11. M. J. Edwards, P. Talelli, J. C. Rothwell, Clinical applications of transcranial magnetic stimulation in patients with movement disorders. Lancet Neurol. 7, 827-840 (2008).

12. J.-P. Lefaucheur, N. André-Obadia, A. Antal, S. S. Ayache, C. Baeken, D. H. Benninger R. M. Cantello, M. Cincotta, M. de Carvalho, D. D. Ridder, H. Devanne, V. D. Lazzaro, S. R. Filipović, F. C. Hummel, S. K. Jääskeläinen, V. K. Kimiskidis, G. Koch, B. Langguth, T. Nyffeler, A. Oliviero, F. Padberg, E. Poulet, S. Rossi, P. M. Rossini, J. C. Rothwell, C. Schönfeldt-Lecuona, H. R. Siebner, C. W. Slotema, C. J. Stagg, J. Valls-Sole, U. Ziemann, W. Paulus, L. GarciaLarrea, Evidence-based guidelines on the therapeutic use of repetitive transcranial magnetic stimulation (rTMS). Clin. Neurophysiol. 125, 2150-2206 (2014).

13. E. M. Wassermann, T. Zimmermann, Transcranial magnetic brain stimulation: Therapeutic promises and scientific gaps. Pharmacol. Ther. 133, 98-107 (2012).

14. U. Ziemann, Transcranial magnetic stimulation at the interface with other techniques: A powerful tool for studying the human cortex. Neuroscientist 17, 368-381 (2011).

15. J. P. Lefaucheur, Repetitive transcranial magnetic stimulation (rTMS): Insights into the treatment of Parkinson's disease by cortical stimulation. Neurophysiol. Clin. 36, 125-133 (2006).

16. M. Hampson, Transcranial magnetic stimulation and connectivity mapping: Tools for studying 
the neural bases of brain disorders. Front. Syst. Neurosci. 4, 40 (2010).

17. G. Thut, T. O. Bergmann, F. Fröhlich, S. R. Soekadar, J.-S. Brittain, A. Valero-Cabré, A. T. Sack, C. Miniussi, A. Antal, H. R. Siebner, U. Ziemann, C. S. Herrmann, Guiding transcranial brain stimulation by EEG/MEG to interact with ongoing brain activity and associated functions: A position paper. Clin. Neurophysiol. 128, 843-857 (2017)

18. N. A. Noh, G. Fuggetta, Human cortical theta reactivity to high-frequency repetitive transcranial magnetic stimulation. Hum. Brain Mapp. 33, 2224-2237 (2012).

19. D. Brignani, P. Manganotti, P. M. Rossini, C. Miniussi, Modulation of cortical oscillatory activity during transcranial magnetic stimulation. Hum. Brain Mapp. 29, 603-612 (2008).

20. G. Fuggetta, N. A. Noh, A neurophysiological insight into the potential link between transcranial magnetic stimulation, thalamocortical dysrhythmia and neuropsychiatric disorders. Exp. Neurol. 245, 87-95 (2013).

21. G. Fuggetta, E. F. Pavone, A. Fiaschi, P. Manganotti, Acute modulation of cortical oscillatory activities during short trains of high-frequency repetitive transcranial magnetic stimulation of the human motor cortex: A combined EEG and TMS study. Hum. Brain Mapp. 29, 1-13 (2008).

22. D. Veniero, D. Brignani, G. Thut, C. Miniussi, Alpha-generation as basic response-signature to transcranial magnetic stimulation (TMS) targeting the human resting motor cortex: $A$ TMS/EEG co-registration study. Psychophysiology 48, 1381-1389 (2011).

23. M. S. Barr, F. Farzan, P. M. Rusjan, R. Chen, P. B. Fitzgerald, Z. J. Daskalakis, Potentiation of gamma oscillatory activity through repetitive transcranial magnetic stimulation of the dorsolateral prefrontal cortex. Neuropsychopharmacology 34, 2359-2367 (2009).

24. G. Thut, D. Veniero, V. Romei, C. Miniussi, P. Schyns, J. Gross, Rhythmic TMS causes local entrainment of natural oscillatory signatures. Curr. Biol. 21, 1176-1185 (2011).

25. G. Thut, C. Miniussi, New insights into rhythmic brain activity from TMS-EEG studies. Trends Cogn. Sci. 13, 182-189 (2009).

26. A. Beuter, J.-P. Lefaucheur, J. Modolo, Closed-loop cortical neuromodulation in Parkinson's disease: An alternative to deep brain stimulation? Clin. Neurophysiol. 125, 874-885 (2014).

27. A. D. Wu, F. Fregni, D. K. Simon, C. Deblieck, A. Pascual-Leone, Noninvasive brain stimulation for Parkinson's disease and dystonia. Neurotherapeutics 5, 345-361 (2008).

28. G. Fuggetta, A. Fiaschi, P. Manganotti, Modulation of cortical oscillatory activities induced by varying single-pulse transcranial magnetic stimulation intensity over the left primary motor area: A combined EEG and TMS study. Neurolmage 27, 896-908 (2005)

29. A. P. Strafella, J. H. Ko, J. Grant, M. Fraraccio, O. Monchi, Corticostriatal functional interactions in Parkinson's disease: A rTMS/[11C]raclopride PET study. Eur. J. Neurosci. 22, 2946-2952 (2005).

30. A. P. Strafella, T. Paus, J. Barrett, A. Dagher, Repetitive transcranial magnetic stimulation of the human prefrontal cortex induces dopamine release in the caudate nucleus. J. Neurosci. 21, RC157 (2001).

31. A. P. Strafella, Striatal dopamine release induced by repetitive transcranial magnetic stimulation of the human motor cortex. Brain 126, 2609-2615 (2003).

32. B. T. Klassen, J. G. Hentz, H. A. Shill, E. Driver-Dunckley, V. G. H. Evidente, M. N. Sabbagh, C. H. Adler, J. N. Caviness, Quantitative EEG as a predictive biomarker for Parkinson disease dementia. Neurology 77, 118-124 (2011).

33. T. S. Rajan, M. F. M. Ghilardi, H.-Y. Wang, E. Mazzon, P. Bramanti, D. Restivo, A. Quartarone, Mechanism of action for rTMS: A working hypothesis based on animal studies. Front. Physiol. 8, 457 (2017).

34. A. Quartarone, H. R. Siebner, J. C. Rothwell, Task-specific hand dystonia: Can too much plasticity be bad for you? Trends Neurosci. 29, 192-199 (2006).
35. U. Ziemann, W. Paulus, M. A. Nitsche, A. Pascual-Leone, W. D. Byblow, A. Berardelli, H. R. Siebner, J. Classen, L. G. Cohen, J. C. Rothwell, Consensus: Motor cortex plasticity protocols. Brain Stimul. 1, 164-182 (2008).

36. S. W. Hughes, M. Lörincz, D. W. Cope, K. L. Blethyn, K. A. Kékesi, H. R. Parri, G. Juhász $\mathrm{V}$. Crunelli, Synchronized oscillations at $\alpha$ and $\theta$ frequencies in the lateral geniculate nucleus. Neuron 42, 253-268 (2004)

37. Y. Zhang, R. Llinas, J. Lisman, Inhibition of NMDARs in the nucleus reticularis of the thalamus produces delta frequency bursting. Front. Neural Circuits 3, 20 (2009).

38. F. Fregni, A. Pascual-Leone, Technology insight: Noninvasive brain stimulation in neurologyperspectives on the therapeutic potential of rTMS and tDCS. Nat. Clin. Pract. Neurol. 3 383-393 (2007).

39. J. Y. Kim, E. J. Chung, W. Y. Lee, H. Y. Shin, G. H. Lee, Y. S. Choe, Y. Choi, B. J. Kim, Therapeutic effect of repetitive transcranial magnetic stimulation in Parkinson's disease: Analysis of [11C] raclopride PET study. Mov. Disord. 23, 207-211 (2008).

40. Y. D. V. D. Werf, A. F. Sadikot, A. P. Strafella, T. Paus, The neural response to transcrania magnetic stimulation of the human motor cortex. II. Thalamocortical contributions. Exp. Brain Res. 175, 246-255 (2006).

41. A. F. Leuchter, I. A. Cook, Y. Jin, B. Phillips, The relationship between brain oscillatory activity and therapeutic effectiveness of transcranial magnetic stimulation in the treatment of major depressive disorder. Front. Human Neurosci. 7, 37 (2013).

42. F. Duecker, A. T. Sack, Rethinking the role of sham TMS. Front. Psychol. 26, 210 (2015).

43. M. F. Folstein, S. E. Folstein, P. R. Mchugh, Mini-mental state: A practical method for grading the cognitive state of patients for the clinician. J. Psychiatr. Res. 12, 189-198 (1975).

44. Movement Disorder Society Task Force on Rating Scales for Parkinson's Disease, The unified Parkinson's disease rating scale (UPDRS): Status and recommendations. Mov. Disord. 18, 738750 (2003).

45. J. Jankovic, M. McDermott, J. Carter, S. Gauthier, C. Goetz, L. Golbe, S. Huber, W. Koller C. Olanow, I. Shoulson, M. Stern, C. Tanner, W. Weiner, Variable expression of Parkinson's disease: A base-line analysis of the DAT ATOP cohort. Neurology 40, 1529 (1990).

46. G. Fuggetta, M. A. Bennett, P. A. Duke, A. M. Young, Quantitative electroencephalography as a biomarker for proneness toward developing psychosis. Schizophr. Res. 153, 68-77 (2014).

47. P. M. Rossini, A. T. Barker, A. Berardelli, M. D. Caramia, G. Caruso, R. Q. Cracco, M. R. Dimitrijević, M. Hallett, Y. Katayama, C. H. Lücking, A. M. de Noordhout, C. D. Marsden, N. M. F. Murray, J. C. Rothwell, M. Swash, C. Tomberg, Non-invasive electrical and magnetic stimulation of the brain, spinal cord and roots: Basic principles and procedures for routine clinical application. Report of an IFCN committee. Electroencephalogr. Clin. Neurophysiol. 91, 79-92 (1994).

48. Y. Oin, P. X U, D. Yao, A comparative study of different references for EEG default mode network: The use of the infinity reference. Clin. Neurophysiol. 121, 1981-1991 (2020).

Acknowledgments: Funding: This research was self funded. Author contributions: G. Fuggetta was responsible for all aspects of obtaining ethics approval, study design, data collection, experimental methods, signal analyses, statistical analysis, data interpretation and manuscript preparation. M. Sandrini was responsible for data interpretation and manuscript preparation. C. Arcaro participated directly in data collection. M. Tinazzi and P. Manganotti were responsible for obtaining ethics approval and recruitment of patients. Competing interests: All authors declare that they have no competing interests. Data and materials availability: All data and materials are available upon request to the corresponding author. 